\title{
Examining the use of process evaluations of randomised controlled trials of complex interventions addressing chronic disease in primary health care-a systematic review protocol
}

Hueiming Liu*, Janini Muhunthan, Adina Hayek, Maree Hackett, Tracey-Lea Laba, David Peiris and Stephen Jan

\begin{abstract}
Background: Randomised controlled trials (RCTs) of complex interventions in primary health care (PHC) are needed to provide evidence-based programmes to achieve the Declaration of Alma Ata goal of making PHC equitable, accessible and universal and to effectively address the rising burden from chronic disease. Process evaluations of these RCTs can provide insight into the causal mechanisms of complex interventions, the contextual factors, and inform as to whether an intervention is ineffective due to implementation failure or failure of the intervention itself. To build on this emerging body of work, we aim to consolidate the methodology and methods from process evaluations of complex interventions in PHC and their findings of facilitators and barriers to intervention implementation in this important area of health service delivery.
\end{abstract}

Methods: Systematic review of process evaluations of randomised controlled trials of complex interventions which address prevalent major chronic diseases in PHC settings. Published process evaluations of RCTs will be identified through database and clinical trial registry searches and contact with authors. Data from each study will be extracted by two reviewers using standardised forms. Data extracted include descriptive items about (1) the RCT, (2) about the process evaluations (such as methods, theories, risk of bias, analysis of process and outcome data, strengths and limitations) and (3) any stated barriers and facilitators to conducting complex interventions. A narrative synthesis of the findings will be presented.

Discussion: Process evaluation findings are valuable in determining whether a complex intervention should be scaled up or modified for other contexts. Publishing this protocol serves to encourage transparency in the reporting of our synthesis of current literature on how process evaluations have been conducted thus far and a deeper understanding of potential challenges and solutions to aid in the implementation of effective interventions in PHC beyond the research setting.

Systematic review registration: PROSPERO CRD42016035572

Keywords: Process evaluations, Primary health care, Complex interventions, Systematic review, Chronic disease, Qualitative

\footnotetext{
* Correspondence: hliu@georgeinstitute.org.au

The George Institute for Global Health, University of Sydney, Level 10, King

George V Building, 83-117 Missenden Rd, PO Box M201, Camperdown, NSW

2050, Australia
} 


\section{Background}

\section{Why is this field of research important?}

With a rapidly rising global burden of disease attributed to non-communicable diseases, access to high quality primary health care (PHC) is essential. Complex interventions, defined as 'interventions that comprise multiple interacting components, although additional dimensions of complexity include the difficulty of their implementation and the number of organisational levels they target, are frequently deployed in an attempt to address health system deficiencies experienced by patients and providers [1]. Choosing a study design to assess effectiveness of complex interventions is not straightforward, and it is recommended to consider randomisation to prevent selection bias and provide robust evidence [2, 3]. Process evaluations, which are typically carried out in conjunction with randomised controlled trials of such interventions, can help explain for whom, how and why a complex intervention had a particular impact [4].

Such evaluations address the question 'Is this intervention acceptable, effective, affordable and feasible (for me or) for this population?' [5]. Process evaluations can enable patient-centred care by providing the opportunity for often over-looked patients' perspectives to be considered. As an example, while a pragmatic trial of a cardiovascular polypill in Australian PHC indicated the polypill was an effective, cost-effective strategy for improving patient adherence and the prescribing of indicated medications, our process evaluation interviews found that clinicians need to consider the polypill strategy alongside other evidence-based strategies. These strategies should cater to specific patient factors such as health literacy, sense of well-being, financial considerations, establishing ongoing respectful clinician and patient relationships and improving accessibility to health care [6].

Despite the generation of good quality evidence, this often does not translate into improved health outcomes [7]. A key barrier in the literature to research translation is cost at different levels, e.g. high outpatient costs for screening to the patient or cost of medications for the programme [8-10]. While health economic evaluations are increasingly being conducted as separate studies to provide evidence of cost-effectiveness to decision makers, there may be cost information that is relevant to the objectives of a process which needs to be investigated. For example, minimising indirect costs to patients is something that is important in understanding why an intervention may be more acceptable to patients compared to standard care. Conversely, for some, indirect costs associated with the intervention may discourage patients from seeking care. These are economic issues which we propose would be important to capture as part of process evaluations but are not strictly captured in health economic evaluations assessing the incremental cost-effectiveness of interventions. It would be pertinent as part of a process evaluation to incorporate relevant cost data from the onset, especially within PHC trials in low- and middleincome countries (LMIC) and in populations which have complex needs and limited funding to be allocated [9]. This would be important as part of a process evaluation, to unpack whether for whom and how an intervention can be implemented into routine practice after the trial is completed. These findings from process evaluations can then inform adoption of interventions into practice and thus the scalability and sustainability of interventions [11].

\section{What is known about this field currently?}

Process evaluation methodology is evolving [4]. Process evaluations were previously synonymous with qualitative research alongside trials and were conducted to provide a deeper understanding of the disease condition, implementation issues and mechanisms of the intervention [12]. However, there is a growing recognition that using qualitative and quantitative data (mixed methods) can help facilitate trial implementation and research translation [13-15]. For instance, stratifying quantitative outcome data by socio-economic status and triangulating it with qualitative interviews, multi-level modelling and embedded cost-analysis in a process evaluation may be useful in determining the relevance and feasibility of a proven effective complex intervention. Using mixed methods, a clearer picture of the intervention may emerge that could aid various stakeholders in their decision-making.

Although 'one size fits all' methods or methodologies are not available, various theories or frameworks to enhance implementation research have been used by researchers to assist in their process evaluations. In early 2015, guidance was published by the Medical Research Council (MRC) UK about the planning, conduct and reporting of process evaluations to aid researchers, policy makers and funders [11]. The article described the proposed functions of process evaluations of looking at feasibility and piloting, evaluation of effectiveness and implementation post-evaluation during the different stages of the development, evaluation and implementation of a complex intervention. These functions expanded upon the conventional definition of process evaluations being limited to during trial implementation and defined 'implementation' as 'the process through which interventions are delivered, and what is delivered in practice'. For example, during the post-evaluation implementation stage, the authors recommend that the process evaluation serves to explore how there is 'routinisation of the intervention into new contexts, and long term implementation/maintenance'. The authors suggest that this function of the process evaluation is needed as reviews have showed that post-trial, complex interventions are only partially maintained. Key recommendations regarding the planning, 
design and conduct, analysis and reporting of process evaluations were also discussed in the MRC recommendations $[4,11]$. For example, arguments for whether there should be a separation or integration of the process evaluation and outcome evaluation teams were presented. The need to integrate process and outcome data in the analysis and the timing of when process data should be analysed in relation to outcome data were discussed.

The appraisal of the quality of process evaluations has not been straightforward partly because of the variability in methods [11, 16, 17]. Grant et al. in a literature review found that the process evaluations were of poor and inconsistent quality and proposed seven criteria for the reporting of process evaluations including clearly labelling that it is a process evaluation [17]. Other suggestions include appraising the quality of the process evaluation based on the methods used. Given that most process evaluations will have a qualitative component, a set of criteria to examine the quality in the reporting of qualitative research will be relevant to most process evaluations $[18,19]$.

Dissemination and reporting of findings from process evaluations especially in academic publications can also be difficult due to a variety of reasons including feasibility due to limited resources for research projects, lag time till dissemination of result or publication bias as usually positive outcome trials will be reported but not necessary negative trials $[11,20]$. This in turn could limit the likelihood of such relevant findings affecting policy and practice.

\section{Why do this review?}

The George Institute for Global Health has a current programme of research which focuses on addressing NCDs through cost-effective and equitable strategies in primary health care settings including LMIC, and with indigenous populations [21]. Our studies trial complex interventions such as capacity-building initiatives with local providers [22], use of innovative mobile technology [23], and cost-effective generic medications (e.g. polypill) within primary health care settings [24]. We have found that at times, despite acceptability and effectiveness of these strategies, there are significant challenges that impact upon their scale up. These barriers could be cultural, political or institutional factors [25], but an important reason for limited translation seems to lie in the lack of understanding of implementation issues within contextual factors for the different stakeholders (e.g. patient, provider, policy makers). For example, while a trial in India of a clinical decision support system on a mobile tablet improved initial diagnosis and antihypertensive management of trial patients, and was deemed acceptable by end-users, only $35 \%$ of patients attended the scheduled 1-month follow-up [23]. Interviews with stakeholders found that limited patient accessibility to medicines and doctors (for a variety of reasons including inadequate staffing, limited primary health care infrastructure) as the key barrier which needs to be overcome. This contrasts with other trials of electronic health tools (e.g. decision support, text messages) in Australia which tend towards generally more positive and sustained results as such presumably because system issues were less of a significant barrier given the universal and subsidised health care available [26-28]. Given the greater burden of early mortality from NCD in LMIC and disadvantaged populations [29], consolidating our findings in this proposed systematic review with an equity-focused lens to better understand how to strengthen PHC within relevant contextual policy and system issues would be useful. Indeed, systematic reviews of interventions in primary health care have concluded that in addition to clinical outcomes, rigorous evaluations of implementation outcomes (e.g. through process evaluations) are needed to ensure changes in practice [30, 31]. We hope that this systematic review will add to the process evaluation methodology and understanding of effective implementation strategies in different PHC settings [32, 33].

\section{Objectives and key questions}

To our knowledge, this is the first systematic review of process evaluations of randomised controlled trial (RCTs) in complex interventions in PHC. For complex interventions, the pre-specification of a theory for how an intervention is expected to work can be highly informative in identifying the mechanisms by which an intervention was hypothesised to have an impact and why it was found to be successful (or not). It provides a framework for assessing the behaviour of individual actors in the implementation of an intervention, potential breakdowns in the interactions between parties and puts into context these actions. Thus, findings from process evaluations from both positive and negative trials can shed light upon implementation facilitators and barriers, which would add to the collective lessons for researchers. Moreover, given that there are numerous theories and frameworks in this area, we thought it would be informative to describe the breadth of methods used and to make some recommendations on evaluation methods that should be incorporated into PEs of complex interventions. Thus, we aim to consolidate the methodology and methods from process evaluations of complex interventions in PHC and their findings of facilitators and barriers to intervention implementation in this important area of health service delivery.

These objectives will be achieved through addressing these questions: (a) Is there and what is the explicit theory behind the conducted process evaluations? (e.g. normalisation process theory, realist framework); (b) What are 
the methods used in these process evaluations? (e.g. qualitative research through semi-structured interviews, surveys); (c) At what stage is the process evaluation done? (i.e. feasibility and piloting, evaluation of effectiveness, or post-evaluation implementation.); (d) If an aim is stated (i.e. in the evaluation of effectiveness stage), how are the results of the RCT integrated with the findings from the process evaluations?; (e) What are the strengths, limitations and potential solutions identified by the authors in conducting the process evaluations?; and (f) What are the barriers and facilitators to the implementation of complex interventions identified by the authors?

\section{Methods/design}

This systematic review will focus on process evaluations of RCTs of complex interventions addressing chronic disease in PHC. We have described our methods as per Preferred Reporting Items for Systematic Review and Meta-Analysis for protocol (PRISMA-P) recommendations, and this checklist is included as an Additional file 1 [34].

\section{Eligibility criteria}

Definitions as per PICO-D have been adapted for the purpose of this review [20,35]:

Participants-participants include patients and health providers in the PHC setting addressing the prevalent chronic diseases as defined by the World Health Organisation-cardiovascular disease, chronic kidney disease, chronic respiratory disease, type 2 diabetes mellitus and depression. PHC as defined by the Alma-Ata declaration [36] as health services provided within the community setting by doctors, nurses and allied health with the goal to achieve better health for all through reforms in universal coverage, public policy, service delivery and leadership [37].

'Intervention'-complex interventions defined as those 'interventions that comprise multiple interacting components, although additional dimensions of complexity include the difficulty of their implementation and the number of organisational levels they target' within PHC [4]. This includes a single-faceted intervention that requires multiple actors or pathways and thus makes the implementation complex. It is envisaged that the complex interventions for chronic diseases (if not explicitly defined as a complex intervention) will have elements of the Wagner chronic care model such as community support, case management, self-management, facilitated family support, organisational change, delivery system design, decision support for health care providers and clinical information systems [38].

Comparator-not applicable

'Outcomes'-(1) findings from the process evaluations of stated implementation barriers and facilitators to the complex intervention. (2) The stated strengths and limitations of the process evaluation methodology from the perspectives of the authors. Both findings will be useful for future conduct of complex interventions in $\mathrm{PHC}$ in the planning, conduct of process evaluations and in the consideration of intervention implementation and what barriers need to be overcome in different PHC settings.

Timing-years of search from 1998. This was chosen because a systematic review by Davies et al. shows that there was poor use of theory in implementation research until at least 1998 [39].

Design-process evaluations of randomised controlled trials of complex interventions in PHC. Process evaluation as defined by 'a study which aims to understand the functioning of an intervention, by examining implementation, mechanisms of impact, and contextual factors' [11]. As discussed by Grant et al., because process evaluations are not clearly labelled as such, qualitative research conducted alongside RCTs with similar aims will be included $[17,40]$.

Exclusion criteria-articles were excluded if they were not a journal article, not a report based on empirical research (e.g. protocol, editorial), not reported in English and reviews and not human research.

\section{Search strategy \\ Information sources}

Databases reporting academic publications (MEDLINE, SCOPUS, PsychInfo, CINAHL, EMBASE, Global Health.) In order to locate any process evaluations whose findings were not published or missed in the database searches, we will search major clinical trial registries for completed process evaluations (e.g. Cochrane Central Registry of Controlled Trials, EU registry, ANZTRN and clinical trial registry (USA)). Authors will be contacted in regard to the outcomes of the RCT and findings of their completed process evaluations.

A search strategy was developed and adapted for each database with the initial support of a medical research librarian. Search terms were based on the review objectives and early scoping searches (see Additional file 2: search strategy), key words: process evaluations (including programme evaluation, qualitative research), complex intervention (including chronic care model and its components of community support, case management, selfmanagement, facilitated family support, organisational change, delivery system design, decision support for health care providers and clinical information systems), randomised controlled trials, PHC (including family practice, general practitioners) and chronic disease (including cardiovascular disease, chronic kidney disease, chronic respiratory disease, type 2 diabetes mellitus and depression).

\section{Study records}

Data management

After the searches, the shortlisted articles will be exported to Endnote. Data will be stored in a common file that is 
password protected on the Institute's server that is accessible by the two reviewers. At each stage of the data selection process during the review (e.g. after consolidation of all articles prior to assessing eligibility based on title and abstract), back up files of the endnote database will be made in order to retrace any steps as needed in the review process, and for any third party adjudication.

\section{Selection process}

Two reviewers will screen all titles and abstracts identifying potential eligible studies based on inclusion and exclusion criteria, and duplicates are to be removed. This will be done independently to reduce the risk of bias. All eligible studies will be retrieved in full text and reviewed by the two reviewers using predesigned eligibility forms (see Additional file 3). Disagreements will be resolved by consensus of a third party in the review team.

\section{Data collection process}

Data from all included studies will be extracted by two reviewers using the eligibility and data extraction forms. The data extraction forms (see Additional file 4) were partly guided by the MRC recommendation for process evaluations and Grant et al.'s suggested minimal factors for reporting on process evaluations [4, 17]. The forms will be pilot tested by the two reviewers on the same three articles, iterative changes will be made when appropriate and the two reviewers will independently extract data from the rest of the included list of articles.

\section{Data items}

Variables to be extracted include data on the RCT and its process evaluation: (1) RCT-study design, setting (rural, urban, country), results (positive, negative or equivalent); (2) process evaluation-any published process evaluation protocol or evidence of pre-specified process evaluation in the main trial protocol, or stated aims of the process evaluation (e.g. examining recruitment, or explaining results), the process evaluation theory, justified methods of integrating trial and process outcomes, stage during which the process evaluation is done (feasibility and piloting, evaluation of effectiveness and post-evaluation implementation), methods of analysis and inclusion of costs incurred.

\section{Outcomes and prioritisation}

The outcomes of interest for our aims are (1) the stated strengths and limitations of the process evaluation methodology from the perspectives of the authors and (2) findings from the process evaluation of stated implementation barriers and facilitators of the complex intervention. Both findings will be useful for future conduct of complex interventions in $\mathrm{PHC}$-in the planning, conduct of process evaluations and when considering the scaling up of an interventions and what barriers need to be overcome in a PHC setting depending on context [1]. For example, the community's need, the type of model or availability of PHC services will be different in developed settings as compared to LMIC.

\section{Risk of bias in individual studies}

For this review, we drew on the use of Tong et al.'s criteria for reporting of qualitative studies [19], on Grant et al.'s proposed framework of minimal requirements for the reporting of process evaluations of cluster randomised controlled trials [17] and on MRC recommendations for process evaluations of complex interventions [4]. Combining insights from these papers, a form of appraisal for risk of bias was derived (see Additional file 5). For the purposes of this review in examining the use of process evaluations alongside RCTs in PHC, studies were not excluded based on quality [20]. Instead, the quality of the studies is presented as a risk of bias graph (low, unclear and high risk) [41].

\section{Data synthesis}

This will involve the aggregation or synthesis of qualitative findings to generate a set of statements that represent that aggregation and categorisation of these findings on the basis of similarity in meaning and contexts. These categories will then be subjected to thematic synthesis in order to produce a single comprehensive set of synthesised findings that can be used as a basis for evidence-based practice. The synthesis of these qualitative data aims to satisfy the criteria established for the reporting of the synthesis of qualitative health research [18]. Abstracted quantitative data (e.g. number of positive trials) will be presented together with a descriptive narrative form including tables and figures to aid in data presentation where appropriate. We will examine how authors address potential bias through a narrative synthesis how well these are reported in the papers and strategies that may have been employed to mitigate this (e.g. triangulation of key findings). Depending on papers included, there may be subgroup analysis of further exploration of any differences of the barriers and facilitators to intervention implementation by context such as indigenous versus non-indigenous and of developed settings as compared to LMIC.

\section{Discussion}

There is a global call for PHC reform in the areas of service delivery, public policy and leadership to enable greater equity and improved health to different populations. To effect this change will require complex interventions involving multiple players (clinicians, community, allied health professionals, policy makers), disciplines (e.g. education, health) and what is successful in one context may not be suitable in another. Process evaluations 
conducted alongside RCTs of complex health interventions are valuable in determining whether a complex intervention should be scaled up or modified for other contexts.

The conduct of process evaluations is still a dynamic area with no clear defined method, partly due to the spectrum of methods (e.g. observation, interviews and routine monitoring data). De Silva et al. in 2014 outlined the integration of the Theory of Change into the MRC framework for complex interventions, and one of its aims was to combine 'process and effectiveness indicators into a single analysis which can help untangle whether, how and why an intervention has an impact in a particular context, and whether it may be suitable for scale up or adaptation for new settings' [42]. Moreover, in regard to future scale up of complex interventions, economic issues pertinent to stakeholders (e.g. patients and providers) would be crucial to policy makers and funders-while this has not been traditionally incorporated together with process evaluations, it would be helpful to see if it has been done [35, 43].

Process evaluations of complex interventions have been increasing in recent years and seem to be variable in objectives, methodology and quality. The MRC guidance in the conduct of process evaluations and in the interpretation of the RCT outcomes may be helpful for researchers to aid in the implementation of effective interventions beyond the research setting. This protocol outlines our methods and design in our efforts to systematically consolidate the collective experience of researchers in this field in conducting, analysing and reporting process evaluations by assembling the findings within the MRC's process evaluation recommendations and to understand previous challenges and potential solutions in the implementation of evidence-based complex interventions in PHC according to context.

\section{Additional files}

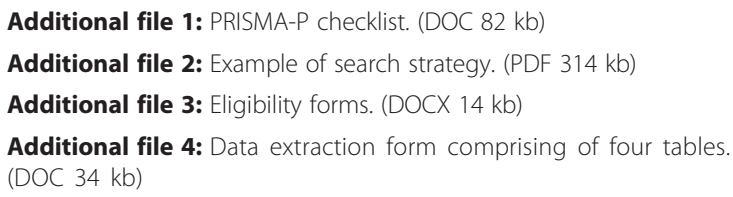

\section{Abbreviations}

LMIC, low- and middle-income countries; MRC, Medical Research Council UK; $\mathrm{PHC}$, primary health care; $\mathrm{RCT}$, randomised controlled trial

\section{Acknowledgements}

Not applicable.

\section{Funding}

This systematic review forms part of HL's PhD thesis and is not externally funded or commissioned.
Availability of data and materials

Not applicable.

\section{Authors' contributions}

HL conceived the study, conducted the scoping searches, designed and piloted the forms and drafted the manuscript; she manages the overall study and will be involved in the study selection, data extraction, synthesis and analysis. JM assisted in the scoping searches, piloted the data and quality appraisal forms, contributed to the drafts of the manuscript and is involved in the study selection. MH provided guidance to $\mathrm{HL}$ in the overall design of the study, assisted in refining the data extraction forms and drafted the manuscript. AH helped revise the manuscript and will contribute to the study selection, data extraction and synthesis. TL contributed to the early drafts of the manuscript, revised the manuscript and will contribute to the study selection. DP contributed to the early drafts of the manuscript and revised the manuscript. SJ conceived the study, provided oversight to $\mathrm{HL}$ and drafted the manuscript. All authors read and approved the manuscript.

\section{Authors' information}

HL had been funded by a University of Sydney Postgraduate scholarship and is currently funded by a National Health and Medical Research Council (NHMRC) scholarship. JM is a recipient of a PhD scholarship from The Australian Prevention Partnership Centre. MH is a recipient of a National Heart Foundation Future Leader Fellowship, Level 2 (100034, 2014-2017). SJ is the recipient of an NHMRC Senior Research Fellowship. TL is the recipient of a NHMRC fellowship. DP is the recipient of a Harkness Fellowship.

\section{Competing interests}

The authors declare that they have no competing interests.

\section{Consent for publication}

Not applicable.

Ethics approval and consent to participate

Not applicable.

Received: 29 April 2016 Accepted: 3 August 2016

Published online: 15 August 2016

\section{References}

1. World Health Organisation. The World Health Report-research for universal health coverage. 2013. available from: http:/www.who.int/whr/2013/report/en/. Accessed 19 Dec 2014.

2. Chalkidou K, Tunis S, Whicher D, Fowler R, Zwarenstein M. The role for pragmatic randomized controlled trials ( $\mathrm{pRCTs}$ ) in comparative effectiveness research. Clin Trials. 2012;9(4):436-46.

3. Craig P, Dieppe P, Macintyre S, Michie S, Nazareth I, Petticrew M, et al. Developing and evaluating complex interventions: the new Medical Research Council guidance. BMJ. 2008;337:a1655. Pubmed Central PMCID: 2769032.

4. Moore GF, Audrey S, Barker M, Bond L, Bonell C, Hardeman W, et al. Process evaluation of complex interventions: Medical Research Council guidance. BMJ. 2015;350:h1258. Pubmed Central PMCID: 4366184.

5. Bonell C, Oakley A, Hargreaves J, Strange V, Rees R. Assessment of generalisability in trials of health interventions: suggested framework and systematic review. BMJ. 2006;333(7563):346-9. Pubmed Central PMCID: 1539056, Epub 2006/08/12. eng.

6. Liu H, Massi L, Laba TL, Peiris D, Usherwood T, Patel A, et al. Patients' and providers' perspectives of a polypill strategy to improve cardiovascular prevention in Australian primary health care: a qualitative study set within a pragmatic randomized, controlled trial. Circ Cardiovasc Qual Outcomes. 2015;8(3):301-8.

7. Tugwell P, Robinson V, Grimshaw J, Santesso N. Systematic reviews and knowledge translation. Bull World Health Organ. 2006;84(8):643-51. Pubmed Central PMCID: 2627444.

8. Tetroe JM, Graham ID, Foy R, Robinson N, Eccles MP, Wensing M, et al. Health research funding agencies' support and promotion of knowledge translation: an international study. Milbank Q. 2008;86(1):125-55. Pubmed Central PMCID: 2690338.

9. Cordero C, Delino R, Jeyaseelan L, Lansang MA, Lozano JM, Kumar S, et al. Funding agencies in low- and middle-income countries: support for knowledge translation. Bull World Health Organ. 2008;86(7):524-34. Pubmed Central PMCID: 2647493. 
10. Evans DB, Edejer TT, Adam T, Lim SS. Methods to assess the costs and health effects of interventions for improving health in developing countries. BMJ. 2005;331(7525):1137-40. Pubmed Central PMCID: 1283282.

11. Moore G AS, Barker M, Bond L, Bonell C, Hardeman W, Moore L, O'Cathain A, Tinati T, Wight D, Baird J. Process evaluation of complex interventions: Medical Research Council guidance. MRC Population Health Science Research Network, London, 2014. Available on: https://www.mrc.ac.uk/documents/pdf/mrc-phsrnprocess-evaluation-guidance-final/. Accessed 22 Jan 2015.

12. Lewin S, Glenton C, Oxman AD. Use of qualitative methods alongside randomised controlled trials of complex healthcare interventions: methodological study. BMJ. 2009;339:b3496. Pubmed Central PMCID: 2741564.

13. Curry LA, Nembhard IM, Bradley EH. Qualitative and mixed methods provide unique contributions to outcomes research. Circulation. 2009;1 19(10):1442-52.

14. Snowdon C. Qualitative and mixed methods research in trials. Trials. 2015; 16:558. Pubmed Central PMCID: 4672490.

15. O'Cathain A, Goode J, Drabble SJ, Thomas KJ, Rudolph A, Hewison J. Getting added value from using qualitative research with randomized controlled trials: a qualitative interview study. Trials. 2014;15:215. Pubmed Central PMCID: 4059032

16. Oakley A, Strange V, Bonell C, Allen E, Stephenson J, Team RS. Process evaluation in randomised controlled trials of complex interventions. BMJ. 2006;332(7538):413-6. Pubmed Central PMCID: 1370978.

17. Grant A, Treweek S, Dreischulte T, Foy R, Guthrie B. Process evaluations for cluster-randomised trials of complex interventions: a proposed framework for design and reporting. Trials. 2013;14(1):15. Pubmed Central PMCID: 3600672. Epub 2013/01/15. eng.

18. Tong A, Flemming K, McInnes E, Oliver S, Craig J. Enhancing transparency in reporting the synthesis of qualitative research: ENTREQ. BMC Med Res Methodol. 2012;12:181. Pubmed Central PMCID: 3552766.

19. Tong A, Sainsbury P, Craig J. Consolidated criteria for reporting qualitative research (COREQ): a 32-item checklist for interviews and focus groups. Int J Qual Health Care. 2007;19(6):349-57.

20. Petticrew M. Time to rethink the systematic review catechism? Moving from 'what works' to 'what happens'. Syst Rev. 2015:4:36.

21. Kirby T. Profile: Australia's George Institute for Global Health. Lancet. 2015:385(9977):1498

22. Alim M, Lindley R, Felix C, Gandhi DB, Verma SJ, Tugnawat DK, et al. Family-led rehabilitation after stroke in India: the ATTEND trial, study protocol for a randomized controlled trial. Trials. 2016;17(1):13. Pubmed Central PMCID: 4704425

23. Praveen D, Patel A, Raghu A, Clifford GD, Maulik PK, Mohammad Abdul A, et al. SMARTHealth India: development and field evaluation of a mobile clinical decision support system for cardiovascular diseases in rural India. JMIR MHealth UHealth. 2014;2(4):e54. Pubmed Central PMCID: 4275493.

24. Laba TL, Hayes A, Lo S, Peiris DP, Usherwood T, Hillis GS, et al. An economic case for a cardiovascular polypill? A cost analysis of the Kanyini GAP trial. Med J Aust. 2014;201(11):671-3.

25. Lawoyin TO, Lawoyin OO. Translation of research into reality in sub-Saharan Africa. Lancet. 2013:381(9884):2146-7.

26. Peiris D, Usherwood T, Panaretto K, Harris M, Hunt J, Redfern J, et al. Effect of a computer-guided, quality improvement program for cardiovascular disease risk management in primary health care: the treatment of cardiovascular risk using electronic decision support cluster-randomized trial. Circ Cardiovasc Qual Outcomes. 2015;8(1):87-95.

27. Neubeck L, Coorey G, Peiris D, Mulley J, Heeley E, Hersch F, et al. Development of an integrated e-health tool for people with, or at high risk of, cardiovascular disease: The Consumer Navigation of Electronic Cardiovascular Tools (CONNECT) web application. International journal of medical informatics. 2016 [Epub ahead of print]

28. Chow CK, Redfern J, Hillis GS, Thakkar J, Santo K, Hackett ML, et al. Effect of lifestyle-focused text messaging on risk factor modification in patients with coronary heart disease: a randomized clinical trial. JAMA 2015;314(12):1255-63.

29. World Health Organisation. Global status of non-communicable diseases World Health Organisation, 2014. Available on: http://www.who.int/nmh/ publications/ncd-status-report-2014/en/. Accessed 25 May 2016.

30. Bero LA, Grilli R, Grimshaw JM, Harvey E, Oxman AD, Thomson MA. Closing the gap between research and practice: an overview of systematic reviews of interventions to promote the implementation of research findings. The Cochrane Effective Practice and Organization of Care Review Group. BMJ. 1998; 317(7156):465-8. Pubmed Central PMCID: 1113716. Epub 1998/08/14. eng.
31. Best A, Greenhalgh T, Lewis S, Saul JE, Carroll S, Bitz J. Large-system transformation in health care: a realist review. Milbank Q. 2012;90(3):421-56. Pubmed Central PMCID: 3479379.

32. Christopher Dye TB, David Evans, Anthony Harries, Christian Lienhardt, Joanne McManus, Tikki Pang, Robert Terry, Rony Zachariah. The world health report 2013: research for universal health coverage. World Health Organisation; 2013. http://www.who.int/whr/2013/report/en/.

33. Gelijns AC, Gabriel SE. Looking beyond translation-integrating clinical research with medical practice. N Engl J Med. 2012;366(18):1659-61.

34. Shamseer L, Moher D, Clarke M, Ghersi D, Liberati A, Petticrew M, et al. Preferred reporting items for systematic review and meta-analysis protocols (PRISMA-P) 2015: elaboration and explanation. BMJ. 2015;349:97647.

35. Anderson LM, Oliver SR, Michie S, Rehfuess E, Noyes J, Shemilt I. Investigating complexity in systematic reviews of interventions by using a spectrum of methods. J Clin Epidemiol. 2013;66(11):1223-9.

36. World Health Organisation. The Declaration of Alma-Ata. 1978. Available from: http://www.who.int/publications/almaata_declaration_en.pdf. Accessed 16 Apr 2015.

37. World Health Organisation. Primary health care [16 april 2015]. Available from: http://www.who.int/topics/primary_health_care/en/. Accessed 25 May 2016.

38. Davy C, Bleasel J, Liu H, Tchan M, Ponniah S, Brown A. Effectiveness of chronic care models: opportunities for improving healthcare practice and health outcomes: a systematic review. BMC Health Serv Res. 2015;15:194. Pubmed Central PMCID: 4448852.

39. Davies P, Walker AE, Grimshaw JM. A systematic review of the use of theory in the design of guideline dissemination and implementation strategies and interpretation of the results of rigorous evaluations. Implement Sci. 2010;5: 14. Pubmed Central PMCID: 2832624, Epub 2010/02/26. eng.

40. Petticrew M, Anderson L, Elder R, Grimshaw J, Hopkins D, Hahn R, et al. Complex interventions and their implications for systematic reviews: a pragmatic approach. Int J Nurs Stud. 2015;52:1211-6.

41. Katikireddi SV, Egan M, Petticrew M. How do systematic reviews incorporate risk of bias assessments into the synthesis of evidence? A methodological study. J Epidemiol Community Health. 2015;69(2):189-95. Pubmed Central PMCID: 4316857.

42. De Silva MJ, Breuer E, Lee L, Asher L, Chowdhary N, Lund C, et al. Theory of change: a theory-driven approach to enhance the Medical Research Council's framework for complex interventions. Trials. 2014;15:267. Pubmed Central PMCID: 4227087

43. Damschroder LJ, Aron DC, Keith RE, Kirsh SR, Alexander JA, Lowery JC. Fostering implementation of health services research findings into practice: a consolidated framework for advancing implementation science. Implement Sci. 2009:4:50. Pubmed Central PMCID: 2736161.

\section{Submit your next manuscript to BioMed Central and we will help you at every step:}

- We accept pre-submission inquiries

- Our selector tool helps you to find the most relevant journal

- We provide round the clock customer support

- Convenient online submission

- Thorough peer review

- Inclusion in PubMed and all major indexing services

- Maximum visibility for your research

Submit your manuscript at www.biomedcentral.com/submit
) Biomed Central 